\title{
Immunoglobulin G4-Related Disease Presented as Recurrent Otitis Media and Mixed Hearing Loss Treated With Cyclophosphamide and Rituximab: A Case Report
}

\author{
Shao SAN ${ }^{1}$, Chia-der $\operatorname{LIN}^{2} \mathbb{D}$, Sheng-ta TSAI ${ }^{3}$, Chao-chun $\operatorname{LIN}^{4} \mathbb{D}$, Po-chang $\mathrm{WU}^{5} \mathbb{C}$ \\ ${ }^{1}$ Department Teaching, China Medical University Hospital, Taichung, Taiwan \\ ${ }^{2}$ Department Otolaryngology, China Medical University Hospital, Taichung, Taiwan \\ ${ }^{3}$ Department Neurology, China Medical University Hospital, Taichung, Taiwan \\ ${ }^{4}$ Department Radiology, China Medical University Hospital, Taichung, Taiwan \\ ${ }^{5}$ Department Rheumatology, China Medical University Hospital, Taichung, Taiwan
}

\begin{abstract}
Immunoglobulin G4-related disease (IgG4-RD) is a systemic autoimmune disease; however, it rarely presents as recurrent otitis media and mixed hearing loss. In this article, we present a 43-year-old male patient who presented with recurrent otitis media and mixed hearing loss that is the $12^{\text {th }}$ case of IgG4-RD with middle and inner ear involvement. We also report the clinical response of cyclophosphamide and rituximab therapy in IgG4-RD. These two drugs have never been used to treat otologic symptoms, but were used to treat other organs affected by IgG4-RD. One year later, the patient underwent mastoidectomy of the right ear and the pathological reports revealed IgG4-related disease. A rheumatologist administered immunosuppressive therapy comprising cyclophosphamide and rituximab. After therapy, patient's right-sided mixed hearing loss partially improved. We recorded all pure tone audiometry data to evaluate the clinical course and treatment effect. Finally, we concluded that pathological confirmation and further immunosuppressive therapy should be considered in a timely manner to prevent hearing impairment. We recommend cyclophosphamide and rituximab for the treatment of diseases involving the middle and inner ear.

Keywords: Cyclophosphamide, hearing loss, immunoglobulin G4-related disease, recurrent otitis media, rituximab.
\end{abstract}

Immunoglobulin G4-related disease (IgG4-RD) is a systemic autoimmune disease, primarily affecting various organ systems such as the pancreas, biliary tree, and liver. The orbit, salivary glands, the thyroid, facial skin, the trigeminal nerve, and the cervical lymph nodes are often affected by IgG4-RD in the head and neck region. ${ }^{1}$ Although the ear is rarely affected, few case reports (we found 11 cases, listed in Table 1) of otologic manifestation can be found in the literature..$^{2-6}$ We excluded one case in Israel which was presented only in the congress. Of the
11 cases, seven were reported in Asia, and all the 11 cases were treated with steroids, one of them with a combination of methotrexate. Herein, we report the $12^{\text {th }}$ case of IgG4-RD involving the middle and inner ear. We treated this patient with cyclophosphamide and rituximab, which have never been used to treat otologic symptoms, but were used to treat other organs affected by IgG4-RD. ${ }^{7-10}$ Pure tone audiometry was recorded serially to define the clinical improvement. In addition, we found that early treatment may lead to better outcomes.

Received: September 13, 2018 Accepted: November 10, 2018 Published online: November 30, 2018

Correspondence: Po-chang Wu, MD. Department Rheumatology, China Medical University Hospital, 40447 Taichung, Taiwan. Tel: +886423910020 e-mail: wpc625531@gmail.com 
Table 1. Immunoglobulin G4-related disease with otologic manifestations, including previously reported cases

\begin{tabular}{|c|c|c|c|c|c|c|}
\hline Otologic symptoms & Case numbers & Country & First author & Journal & Published year & Treatment \\
\hline $\begin{array}{l}\text { Bilateral hearing loss } \\
\text { and ear fullness }\end{array}$ & 1 & Korea & Cho et al. ${ }^{2}$ & $\begin{array}{c}\text { Clinical and } \\
\text { Experimental } \\
\text { Otorhinolaryngology }\end{array}$ & 2011 & $\begin{array}{l}\text { Prednisolone and } \\
\text { methotrexate }\end{array}$ \\
\hline $\begin{array}{l}\text { Sensorineural hearing } \\
\text { loss }\end{array}$ & 3 & Argentina & Gallo et al. ${ }^{3}$ & $\begin{array}{c}\text { Clinical and } \\
\text { Experimental } \\
\text { Otorhinolaryngology }\end{array}$ & 2011 & Glucocorticoids \\
\hline Otitis media & 4 & Japan & Takagi et al. ${ }^{5}$ & $\begin{array}{c}\text { Annals of Otology, } \\
\text { Rhinology \& } \\
\text { Laryngology }\end{array}$ & 2014 & $\begin{array}{c}\text { Oral } \\
\text { prednisolone }\end{array}$ \\
\hline $\begin{array}{l}\text { Sensorineural hearing } \\
\text { loss }\end{array}$ & 1 & Japan & Takagi et al. ${ }^{5}$ & $\begin{array}{c}\text { Annals of Otology, } \\
\text { Rhinology \& } \\
\text { Laryngology }\end{array}$ & 2014 & $\begin{array}{c}\text { Oral } \\
\text { prednisolone }\end{array}$ \\
\hline Hearing loss & 1 & China & Lu et al. ${ }^{4}$ & $\begin{array}{l}\text { Otology and } \\
\text { Neurotology }\end{array}$ & 2017 & Glucocorticoids \\
\hline Otitis media & 1 & Australia & Wuesthoff et al. ${ }^{6}$ & $\begin{array}{l}\text { SAGE Open Medical } \\
\text { Case Reports }\end{array}$ & 2018 & Glucocorticoids \\
\hline
\end{tabular}

\section{CASE REPORT}

A 43-year-old male patient complained of right ear fullness that persisted for two years; he did not have type II diabetes mellitus, hypertension, or cholesteatoma. He was diagnosed to have otitis media with effusion; status post tympanocentesis was performed at another hospital at three

(a)

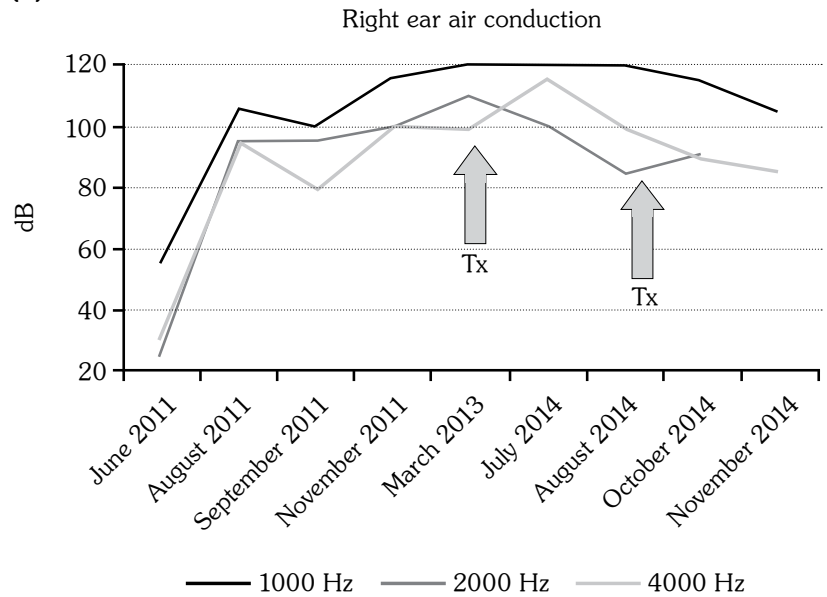

different times; however, he suffered relapse soon after each treatment procedure. He then visited local medical doctors several times for tympanocentesis. In August 2011, he developed right hearing impairment for which he visited our hospital. An ear-nose-throat specialist confirmed right severe mixed hearing loss by pure tone audiometry (Figure 1). Mastoid computed (b)

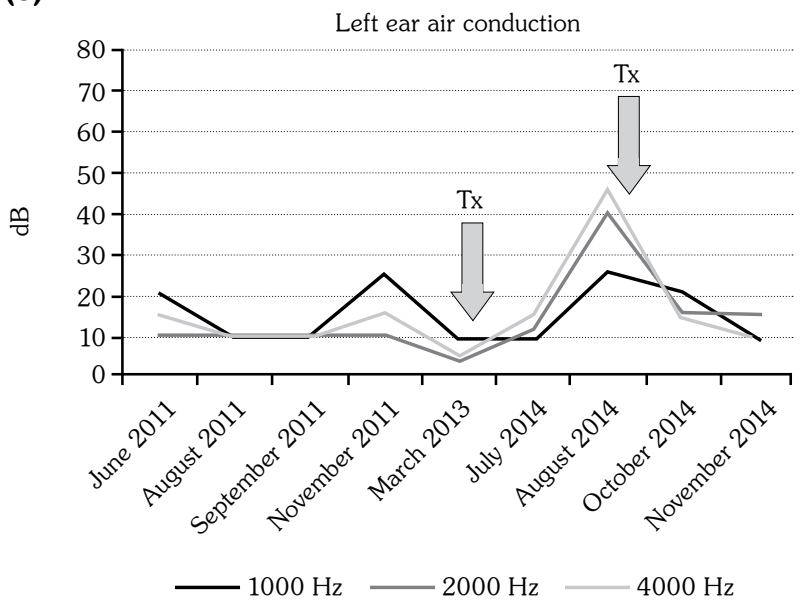

Figure 1. (a) Pure tone audiometry of right ear air conduction at 1000, 2000, and $4000 \mathrm{~Hz}$. X-axis indicates time we performed pure tone audiometry; "Jun-11" implies June 2011. Y-axis indicates lowest decibels (dB) that patient can hear. "Tx" indicates time we gave him treatment; one yellow arrow represents three doses of cyclophosphamide and two doses of rituximab, two weeks between each doses. (b) Left ear air conduction is normal initially. According to World Health Organization, hearing impairment is defined by thresholds $>25 \mathrm{~dB}$. However, threshold increased to $>25 \mathrm{~dB}$ in July 2014 and then decreased to normal range after second course of treatment. 


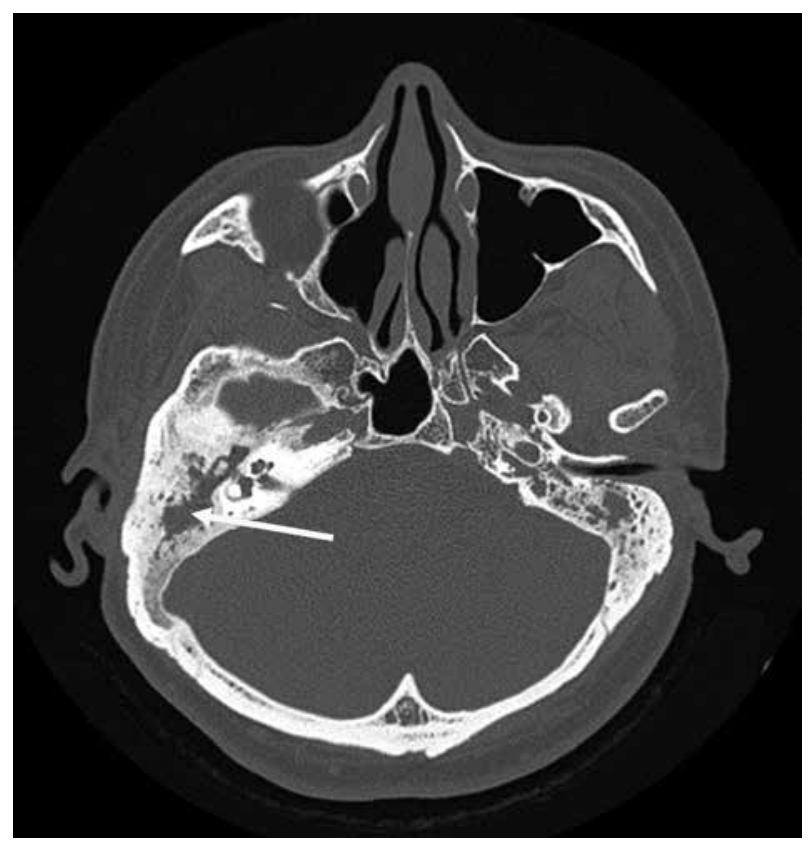

Figure 2. Mastoid computed tomography with bone window showing abnormal soft tissue infiltration in right middle ear cavity and right mastoid process with erosion of ossicular chain (arrow).

tomography $(\mathrm{CT})$ revealed abnormal soft tissue infiltration in his right middle ear cavity and right mastoid process with bony destruction (Figure 2). He received right tympanomastoidectomy in
September 2011. His mastoid cavity was filled with granulation tissue and pus. His pathology report showed rich plasmacytoid cell infiltration. In the following two years, the patient did not visit our hospital, but he received several treatments for his ear from local medical doctors. His hearing, however, did not improve. Furthermore, the patient experienced an episode of seizure in January 2013. Magnetic resonance imaging of the brain revealed $T_{2}$ hyperintensity and leptomeningeal enhancement (Figure 3), which indicated inflammation of the central nervous system. At this point, he revisited our hospital. Mastoid CT revealed abnormal soft tissue infiltration in his right middle ear cavity and right mastoid process with bony destruction. Hence, right modified mastoidectomy was performed and pulsatile granulomatous mass over his right mastoid cavity was found. His pathology report revealed plasma cell granuloma; however, the possibility of plasmacytoma was excluded on the basis of immunostaining results. In addition, it revealed positive IgG4 stain, with 59 IgG4positive plasma cells in a high-power field (Figure 4). The percentage of IgG4-positive plasma cells in the field was approximately $45 \%$. This finding led to the diagnosis of IgG4-RD, after which we initiated immunotherapy with cyclophosphamide and rituximab from
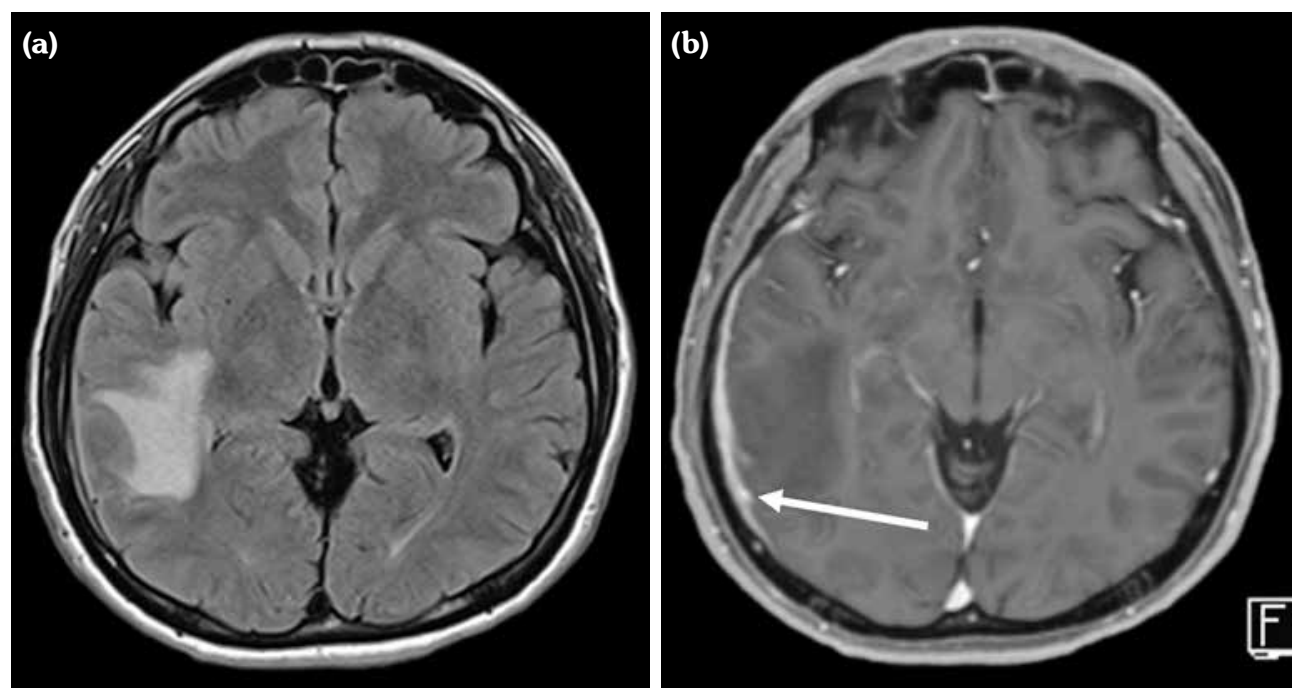

Figure 3. Brain magnetic resonance imaging taken on January 9, 2013, after his seizure episode. (a) $\mathrm{T}_{2}$-W fluid-attenuated inversion recovery axial image revealed abnormal hyperintensity in right temporooccipital lobe, indicating focal edema. (b) Gadolinium-enhanced $\mathrm{T}_{1}-\mathrm{W}$ axial image revealing pachymeningeal enhancement in right temporooccipital region (arrow). 


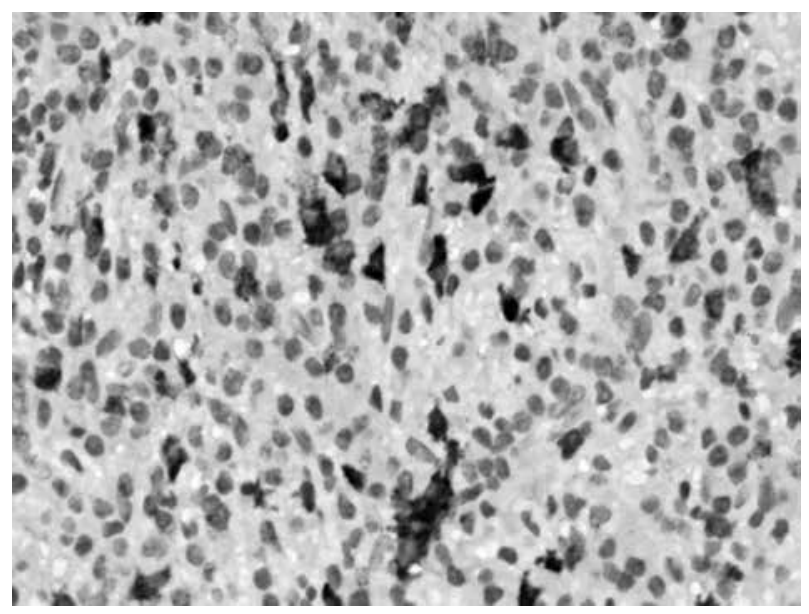

Figure 4. Pathology (immunoglobulin G4 staining, 400×). About 59 immunoglobulin G4-positive plasma cells can be observed in this high-power field (dark parts). Tissue source: a pulsatile granulomatous mass above patient's right mastoid cavity.

April 2013 onward. Subsequently, the patient's symptoms subsided and he did not require as frequent tympanocentesis as he did earlier. The erythrocyte sedimentation rate, IgG, and IgG4 levels also decreased after the treatment. The follow-up mastoid CT revealed remission of the pseudo tumor in his right middle ear cavity (Figure 5). A written informed consent was obtained from the patient.

\section{DISCUSSION}

In this article, we reported the case of a patient with IgG4-RD who had recurrent otitis media and mixed hearing loss. Cho et al. ${ }^{2}$ reported a case of fluctuating mixed-type hearing loss, whereas Gallo et al. ${ }^{3}$ reported three cases of sensorineural hearing loss. Later, Cho et al. ${ }^{11}$ clarified their findings to report that air-bone gap did not improve with ventilation tube insertion and antibiotics during the initial treatment; rather, it improved with immunosuppressive therapy. This outcome indicated that the disease activity of IgG4-RD paralleled in the middle ear and inner ear depending on the immunosuppressive therapy. We confirmed this observation in our patient, in whom the air-bone gap did not improve after middle ear surgery and antibiotics treatment; however, it improved with immunosuppressive therapy comprising cyclophosphamide and rituximab. To the best of our knowledge, autoimmune inner ear disease manifests as progressive, bilateral, often asynchronous, sensorineural hearing loss that can be associated with vestibular symptoms. ${ }^{12}$

Regarding the differential diagnosis, we considered granulomatosis with polyangiitis (Wegener's vasculitis). We assessed the antineutrophil cytoplasmic antibody and pathology results to exclude it.
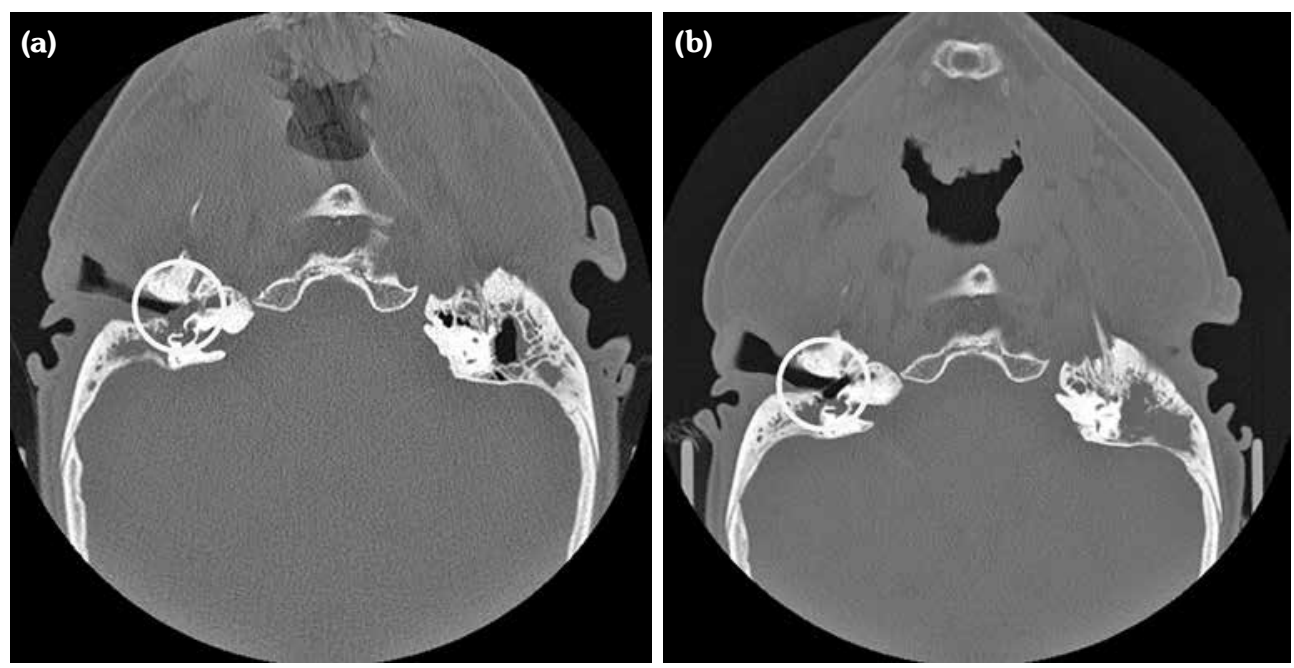

Figure 5. Follow-up mastoid computed tomography in August 2014 after cyclophosphamide and rituximab treatment showing remission of pseudo tumor in right middle ear cavity in comparison with pre-treatment mastoid computed tomography taken three years ago (white circles, (a) August 2011 pre-treatment, (b) August 2014 post-treatment). 
Regarding the patient's left ear, he never complained of fullness sensation or hearing impairment. However, the pure tone audiometry revealed impaired air conduction since July 2014, and the hearing improved after treatment of cyclophosphamide and rituximab in August 2014. Compared with the right ear, we found better improvement in hearing in his left ear (to the normal condition). This observation taught us that earlier immunosuppressive treatment may lead to superior recovery.

Immunoglobulin G4-related disease is rare; however, it can present as recurrent otitis media and mixed hearing loss. We should therefore consider pathological confirmation and further immunosuppressive therapy in a timely manner to prevent hearing impairment in such cases. Moreover, we recommend that an aggressive therapy with glucocorticoids, cyclophosphamide, and rituximab may be useful in treating such patients.

\section{Declaration of conflicting interests}

The authors declared no conflicts of interest with respect to the authorship and/or publication of this article.

\section{Funding}

The authors received no financial support for the research and/or authorship of this article.

\section{REFERENCES}

1. Mulholland GB, Jeffery CC, Satija P, Côté DW. Immunoglobulin G4-related diseases in the head and neck: a systematic review. J Otolaryngol Head Neck Surg 2015;44:24.

2. Cho HK, Lee YJ, Chung JH, Koo JW. Otologic Manifestation in IgG4-Related Systemic Disease. Clin Exp Otorhinolaryngol 2011;4:52-4.

3. Gallo JR, Ortiz AC, Paira SO. IgG4-Related Disease and Sensorineural Hearing Loss. Clin Exp Otorhinolaryngol 2014;7:236-7.

4. Lu P, Sha Y, Wang F, Wang S. IgG4-Related Sclerosing Disease Involving Middle Ear. Otol Neurotol 2017;38:65-7.

5. Takagi D, Nakamaru Y, Fukuda S. Otologic manifestations of immunoglobulin G4-related disease. Ann Otol Rhinol Laryngol 2014;123:420-4.

6. Wuesthoff $\mathrm{C}$, Allende A, Patel N. IgG4 disease of the ear: Report and review. SAGE Open Med Case Rep 2018;6:2050313X18791428.

7. Khosroshahi A, Bloch DB, Deshpande V, Stone $\mathrm{JH}$. Rituximab therapy leads to rapid decline of serum IgG4 levels and prompt clinical improvement in IgG4-related systemic disease. Arthritis Rheum 2010;62:1755-62.

8. Khosroshahi A, Carruthers MN, Deshpande V, Unizony $\mathrm{S}$, Bloch DB, Stone JH. Rituximab for the treatment of IgG4-related disease: lessons from 10 consecutive patients. Medicine (Baltimore) 2012;91:57-66.

9. Khosroshahi A, Stone JH. Treatment approaches to IgG4-related systemic disease. Curr Opin Rheumatol 2011;23:67-71.

10. Wu PC, Tien PT, Li YH, Chen RY, Cho DY. IgG4-related cerebral pseudotumor with perineural spreading along branches of the trigeminal nerves causing compressive optic neuropathy: A case report. Medicine (Baltimore) 2017;96:8709.

11. Cho HK, Lee YJ, Chung JH, Koo JW. In Reply: IgG4 Related Disease and Sensorineural Hearing Loss. Clin Exp Otorhinolaryngol 2014;7:238-9.

12. Buniel MC, Geelan-Hansen K, Weber PC, Tuohy VK. Immunosuppressive therapy for autoimmune inner ear disease. Immunotherapy 2009;1:425-34. 